\title{
NodeI4.0:integrando sistemas legados à indústria 4.0
}

\author{
NodeI4.0, Integrating legacy systems to industry 4.0
}

\author{
Antônio Paulo Batista Junior ${ }^{1}$ (i) http://orcid.org/0000-0002-4773-3442 \\ Sérgio Campello Oliveira ${ }^{1}$ (b) http://orcid.org/0000-0003-1058-1139 \\ ${ }^{1}$ Escola Politécnica de Pernambuco, Universidade de Pernambuco, Recife, Brasil, \\ E-mail do autor principal: Antônio Paulo Batista Juniorapbj1986@gmail.com
}

\section{Resumo}

Ao longo da história a indústria sofreu várias revoluções. Essas revoluções são sempre impulsionadas pela evolução de uma nova tecnologia. Atualmente estamos vivenciando a $4^{a}$ revolução industrial, denominada de indústria 4.0, impulsionada pela internet das coisas. Os fabricantes de equipamentos estão se adequando a essa revolução, fabricando equipamentos que atendem às novas especificações, mas a adequação da indústria às novas especificações exige um alto custo com troca dos equipamentos. O presente trabalho propõem a prototipação do NodeI4.0, um sistema embarcado com características de um CPS (Cyber Physical System) e que tenha a possibilidade de adicionar qualquer sistema legado a um nível smart connection da arquitetura 5C. O NodeI4.0, dependendo do seu modo de operação, envia dados de duas entradas digitais e uma analógica a um servidor e/ou recebe dados (que podem acionar duas saídas digitais e uma analógica)através de rede sem fio IEEE 802.11, utilizando o protocolo http. O protótipo confere a qualquer sistema legado a possibilidade de ter integração com a indústria 4.0.0 protótipo foi testado em um sistema de controle de nível onde foi capaz de realizar um controle descentralizado com velocidade satisfatória para o processo, adicionando a característica de um CPS e integrando esse sistema à indústria 4.0.

Palavras-Chave: Indústria 4.0,Cyber phisical system, Internet das coisas, Sistemas legados.

\section{Abstract}

Throughout history the industry has undergone several revolutions. These revolutions are always driven by the evolution of a new technology. We are currently experiencing the 4th industrial revolution, called industry 4.0, driven by the internet of things. Equipment manufacturers are adapting to this revolution, manufacturing equipment that meets the new specifications, but the suitability of the industry to the new specifications requires a high cost With equipment change. The present work proposes the prototyping of NodeI4.0, an embedded system with characteristics of a CPS (Cyber Physical System) and that has the possibility of adding any legacy system to a smart connection level of the 5C architecture. NodeI4.0, depending on its mode of operation, sends data from two digital and one analog inputs to a server and / or receives data (which can trigger two digital and one analog outputs) over the IEEE 802.11 wireless network, using the Http protocol. The prototype gives any legacy system the possibility of integration with industry 4.0 . The prototype was 
tested in a level control system where it was able to perform a decentralized control with a satisfactory speed for the process, adding the characteristic of a CPS and Integrating this system with industry 4.0.

Keywords: Industry 4.0, Cyber physical System, Internet of Things, Legacy systems.

\section{Introdução}

Observando ao longo da história verificamos que a indústria passou por várias modernizações desde sua concepção na primeira revolução industrial onde,tevese a troca da mão de obra que antes era impulsionada por força humana, por máquinas a vapor. Essa revolução ocasionou mudanças na economia bem como em toda sociedade da época[1].

Sempre que há uma evolução significante de uma determinada tecnologia há também uma mudança drástica no cenário industrial, mudanças essas que foram verificadas na segunda revolução com a utilização da energia elétrica e a implementação de processos industriais baseados em linhas de montagem, subseqüentemente, na terceira com as inovações nos campos da informática e eletrônica e sua aplicação no campo da produção, o surgimento do CLP(Controlador Lógico Programável) controlando processos produtivos.

Hoje, mais uma vez, estamos vivenciando uma revolução industrial, a $4^{a}$ revolução, onde os motivadores são avanços de tecnologias que não eram alcançados a anos atrás, e que tem como pilar central a utilização de IoT(Internet of Things), dados e serviços em poucas palavras essas revolução e a revolução da descentralização.

Os grandes fabricantes de equipamentos estão adequando seus produtos para atender às demandas dessa grande revolução. Visitando o site da empresa Festo[2], com uma rápida busca sobre produtos e soluções para indústria 4.0, observamos essa tendência para os novos produtos. Mais para adequação de todo parque industrial à indústria 4.0 está envolvido um alto custo devido a troca por equipamentos que ainda desempenham suas funções básicas para as quais foram projetados.

Este trabalho tem por objetivo a criação de um sistema embarcado que tenha características de Sistemas Ciber-Físicos que possibilite a essas industrias, compostas por sistemas legados, serem inseridas na $4^{a}$ revolução industrial.

\section{Industria 4.0}

Por definição indústria é a atividade que transforma matéria-prima em produtos comercializáveis e que ao longo da história sofreu algumas revoluções proporcionadas por saltos tecnológicos. Esses saltos levaram ao status atual onde temos uma industria altamente mecanizada e automatizada. A visão futura nos remete a uma industria onde o sistema de produção seja modular, customizado e individual mais mantendo a eficiência e os custos da produção em massa. Essa tendência futura nos coloca no cenário de mais uma revolução industrial, a $4^{a}$, a qual foi rotulada de "Industria $4.0 "[1]$.

A propagação do termo "industria 4.0" origina-se da iniciativa do governo alemão de propor recomendações para uma industria com estratégia high-tech até 2020[2], através de um grupo de trabalho "Industrie 4.0 Working Group" [3].Esse grupo considera a integração dos sistemas IoT como fator chave para a revolução industrial. Ainda mais importante que IoT é a fusão entre mundo físico e virtual conseguido através do CPS (Cyber Physical Systems). CPS é a integração entre processos físicos com a capacidade de controlar e monitorar, normalmente com loop de feedback. A fábrica inteligente proposta pela revolução 4.0 define as fábricas como vivas, pois têm a capacidade de conectar: pessoas, coisas, dados e novas organizações para condução de processos industriais [3].Essas fábricas estão caracterizadas por cinco fatores: Smart networking, Mobility, Flexibility, Integration of customers e New innovative business models[4].

\section{Cyber Physical System}

Cyber physical systems (CPS) são sistemas automatizados que permitem a ligação do mundo 
físico com mundo computacional. Eles têm a capacidade de comunicação com diversos dispositivos e redes, diferentemente do sistema embarcado padrão onde o acesso acontece a um único dispositivo por vez [4].

CPS normalmente tem em sua arquitetura básica uma unidade central de controle, normalmente um microcontrolador que controlam os atuadores e sensores os quais são necessário para interface com mundo físico e ainda contam com uma interface de comunicação para interagir com outros CPS ou para o envio de dados para uma nuvem. A parte mais importante de um CPS é a capacidade de troca de dados através de redes [4].

Como a 40 revolução industrial está no seu início e a utilização do CPS nessa revolução também, ainda não existe uma formalização da arquitetura. Contudo existe uma recomendação segundo Lee [5], que propõe 5 níveis de arquitetura CPS para indústria 4.0 denominados de 5C: Connection, Conversion, Cyber, Cognition, Configure. Esses níveis estão ilustrados na Figura 1.

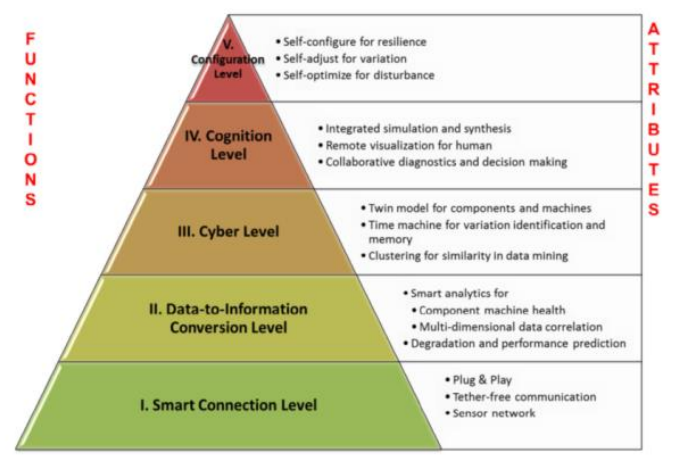

Figura 1: $5 \mathrm{C}$ arquitetura para implementação de um CPS.

\section{Sistemas Legados}

Sistemas legados têm um significado bem abrangente. Para a área de TI, esse termo remete a sistemas antigos e obsoletos de difícil manutenção, mais para a indústria 4.0 podemos pensar em sistemas legados como sistemas existente na indústria que não atendem aos requisitos mínimos para que esses sistemas possam ser integrados a essa nova indústria. Se adotadas as recomendações da arquitetura $5 \mathrm{C}$ para indústria 4.0 qualquer equipamento ou sistema no mínimo precisa atender ao requisito do nível mais básico dessa recomendação, formando assim um CPS de nível smart conection.

Os sistemas legados podem ser de dois tipos digitais e analógicos e obedecem os padrões industriais. Para os que operam de forma digital temos 24V correspondendo a nível lógico 1 e $0 \mathrm{~V}$ a nível lógico 0.Para os que operam de forma analógica existem dois padrões, 0 a $10 \mathrm{~V}$ e 4 a $20 \mathrm{~mA}$ com tensão constante de $24 \mathrm{~V}$. Esses padrões são utilizados para sensores e atuadores. Alguns exemplos são mostrados na Figura 2.

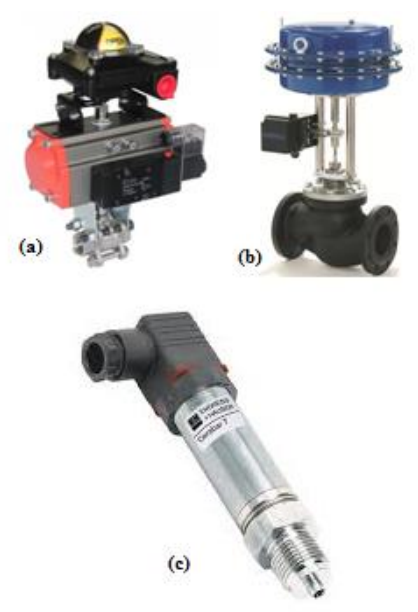

Figura 2: Válvula on/off(a).

Válvula proporcional(b).Sensor de Pressão(c).

Todos esses sensores e atuadores estão presentes em grande parte das indústrias e, com a chegada da $4^{a}$ revolução, se tornam ou compõem sistemas legados.

\section{NodeI4.0}

NodeI4.0 é um protótipo embarcado que tem como características adicionar a qualquer sistema legado o nível Smart conection da arquitetura 5C.

NodeI4.0 é composto de duas entrada digitais, uma entrada analógica, um microcontrolador, um módulo de comunicação padrão IEEE 802.11, duas 
saídas digitais e uma saída analógica, Figura 3.

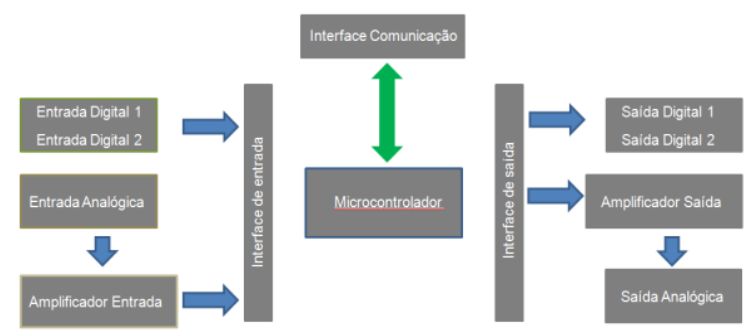

Figura 3: Diagrama de bloco NodeI4.0

\subsection{Entradas digitais}

O protótipo é composto de duas entradas digitais opto-isoladas com padrão de tensão industrial, onde o nível lógico 0 corresponde a $0 \mathrm{~V}$ e nível lógico 1 a $24 \mathrm{~V}$, respectivamente, Figura4.

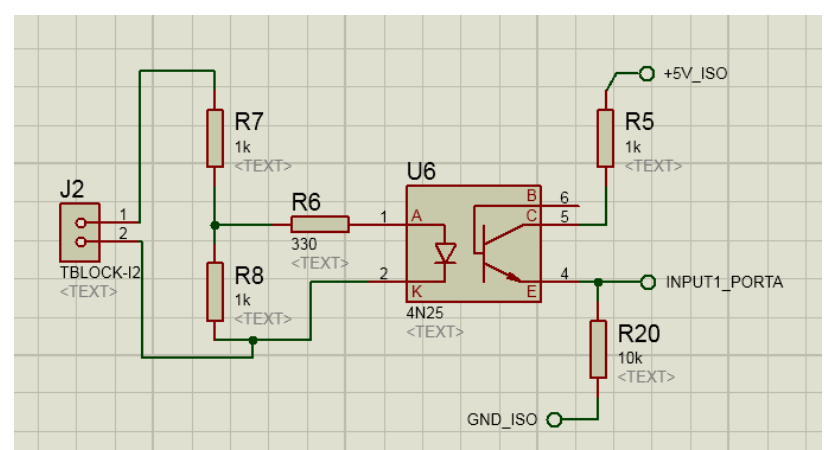

Figura 4: Circuito Entrada Digital

\subsection{Entradas Analógica}

A entrada analógica é composta de um circuito de amplificação para atenuar a tensão analógica dos padrões industriais de 0 a $10 \mathrm{~V}$ para níveis de 0 a $5 \mathrm{~V}$ que possam ser lidos pelo conversor A/D. Nesse protótipo o conversor utilizado é o MCP3201, que é um conversor de 12 Bits com comunicação através de uma interface SPI. O microcontrolador PIC, presente no protótipo, já conta com um conversor $A / D$, mas $O$ fato dele estar integrado ao controlador não permite uma isolação. Com o MCP3201 é possível ter uma isolação através de um barramento optoisolado de alta frequência, Figura 5.

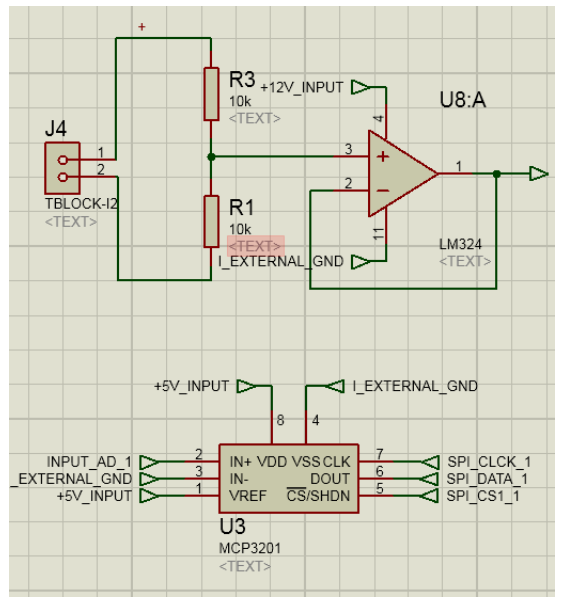

Figura 5: Circuito de Entrada Analógica

\subsection{Microcontrolador}

O microcontrolador escolhido foi 0 PIC18F2550 de 8 bits por ser ideal para aplicações de baixo consumo e conectividade com vários padrões de comunicação. Ele possui memória de programa do tipo flash de 32KB, 2048 bytes de RAM podendo operar com frequência de até $48 \mathrm{Mhz}$. Possui hardware para comunicação SPI e UART que são importantes para o protótipo e está configurado para operar na frequência de $8 \mathrm{Mhz}$.

O código no microcontrolador encapsula e transmite as variáveis proviniente dos I/O através de comunicação serial sobre protocolo customizado, responsável pelo gerenciamento do modos de operação do node.

\subsection{Interface de Comunicação}

Foi escolhido para comunicação o padrão IEEE 802.11 pelo fato de ser comum nas industrias o padrão de comunicação ethernet via cabo (IEEE802.3). Com isso, a implementação de redes wifi tem um custo já reduzido,por se utilizar do padrão ethernet já existente, bastando apenas inserir um roteador e uma antena e devido a isso as limitações físicas de distância são minimizadas. Para atender essas especificações foi escolhido um módulo baseado no sistema integrado em um chip (System-on-Chip - SoC) ESP8266 da Espressif[6].

O SoC contém um processador de 32 bits, camada física do padrão IEEE 802.11 implementada em hardware, periféricos para entradas e saídas digitais, entrada analógica e barramentos de comunicação que podem estar disponíveis dependendo do modelo escolhido. No protótipo, o módulo utilizado foi o ESP-01 [6] que 
possui um pequeno formato e integra, além do SoC, uma memória do tipo flash de $512 \mathrm{~Kb}$ para armazenamento do firmware e dados, Figura 6.

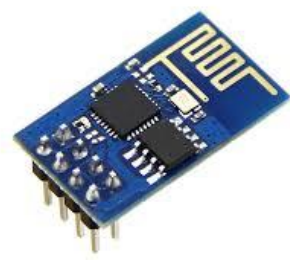

Figura 6: esp8266-01

\subsection{Saídas Digitais}

O protótipo contém duas saídas digitais de coletor aberto que podem atender os padrões de tensão de comando industriais para sinais digitais, podem operar com tensão entre 0 a 24 Volts, com uma corrente constante de $1 \mathrm{~A}$ com picos de $1,5 \mathrm{~A}$.Ambas são opto-isoladas como indicado na figura 7.

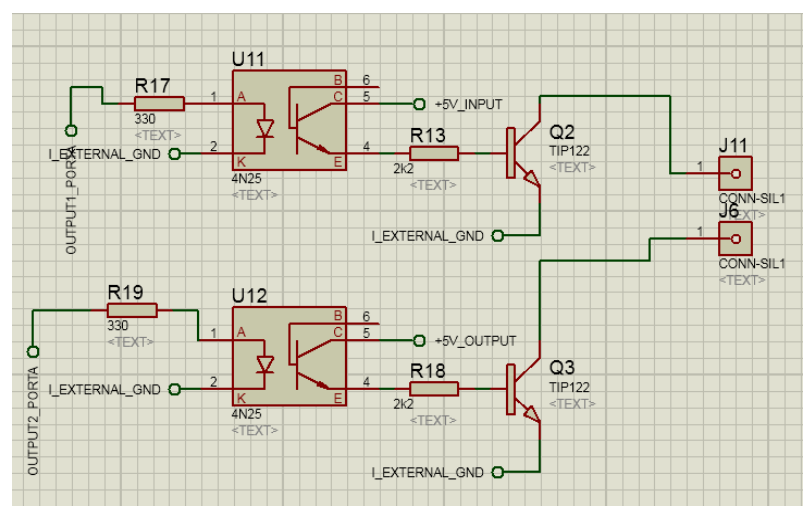

Figura 7: Saídas digitais

\subsection{Saídas Analógicas}

A saída analógica e compostas por um conversor D/A, O MCP4921de 12 bits, com comunicação SPI integrada a um amplificador operacional para amplificar o sinal analógico para níveis industriais, no caso do protótipo de 0 a $10 \mathrm{~V}$, Figura 8.

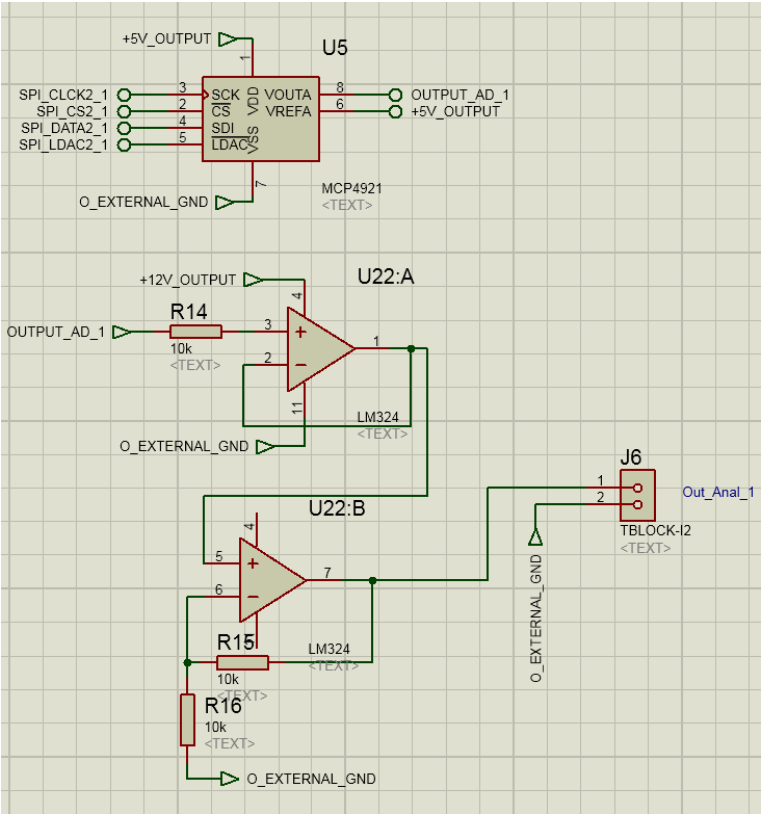

Figura 8: Saídas Analógicas

\subsection{Modos de Operação}

O NodeI4.0 opera em dois modos, acesspoint e station, o primeiro modo é usado apenas para inserir as configurações que serão usadas no modo station. No modo station o NodeI4.0 se conecta a um roteador e envia os dados a um servidor broker através de uma rede wifi. Para configurar o módulo é necessário entrar no modo acesspoint habilitando o jump j14 e j15:

Com Node configurado para modo acesspoint e em seguida reiniciando-o será gerada uma rede wifi com SSID "NodeI4.0". Acessando a rede com a interface de rede do computador em modo DHCP e usando o password correto será possível visualizar a página de configuração digitando no browser o IP "192.168.4.1", na figura 9 temos a tela de configuração e na tabela 1 a descrição de cada campo: 


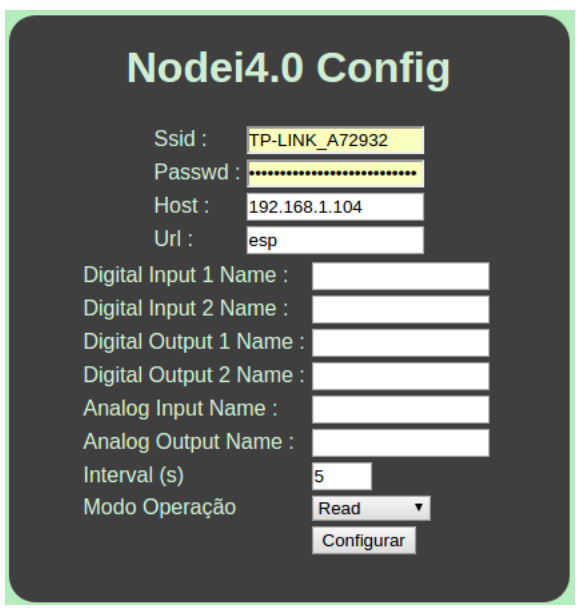

Figura 9: Tela configuração - Modo AP

Tabela 1: Variáveis de configuração modo AP

\begin{tabular}{|c|c|}
\hline Campo & Descrição \\
\hline SSID & $\begin{array}{l}\text { Nome da rede ao qual } \\
\text { se deseja conectar, } \\
\text { uma rede já } \\
\text { configurada em um } \\
\text { roteador. }\end{array}$ \\
\hline Passwd & senha da rede \\
\hline Host & $\begin{array}{l}\text { IP do servidor que irá } \\
\text { receber e tratar os } \\
\text { dados }\end{array}$ \\
\hline Url & $\begin{array}{l}\text { Página para qual os } \\
\text { dados serão enviados }\end{array}$ \\
\hline Interval & $\begin{array}{l}\text { Tempo em que os } \\
\text { dados serão enviados e } \\
\text { recebidos }\end{array}$ \\
\hline Modo Operação & $\begin{array}{l}\text { No modo station } \\
\text { existem três sub } \\
\text { modos de operação } \\
\text { "Read","Write" } \\
\text { "Read/Write", no sub } \\
\text { modo write os dados } \\
\text { apenas são escrito nas } \\
\text { saídas do NodeI4.0, } \\
\text { acionado assim as } \\
\text { saídas digitais e } \\
\text { ajustando o nível de } \\
\text { tensão no conversor } \\
\text { D/A. No sub modo read } \\
\text { as entrada analógicas e } \\
\text { digitais são lidas e } \\
\text { enviadas ao servidor, o } \\
\text { sub modo } \\
\text { "Read/Write" é a }\end{array}$ \\
\hline
\end{tabular}

combinação dos dois modos sendo que a escrita e subsequente a leitura.

O dados provenientes das entradas são enviados ao servidor quando o node está no modo station e submodo Read ou Read/Write através do protocolo HTTP, os valores das entradas digitais e analógicas compõem a URL de requisição, usando a configuração da Figura 9,a URL teria a seguinte composição.

"HTTP://192.168.1.104/esp/eD1/eD2/eA"

Onde eD1, eD2 serão os valores das entradas digitais 1 e 2 respectivamente e eA o valor da entrada analógica. Figura 10 demonstra a URL após requisição.

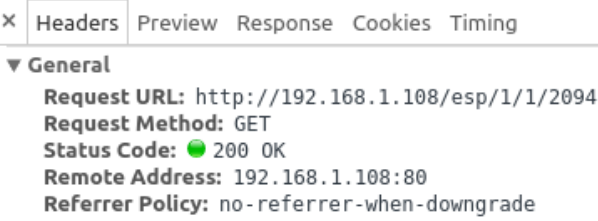

Figura 10 : Requisição HTTP, envio de valores ao servidor

Quando NodeI4.0 está no modo station e submodo Write ou Read/Write a escrita dos valores provenientes do servidor no protótipo se da através da resposta da requisição da mesma requisição HTTP de envio dos dados.Figura 11 resposta da requisição.

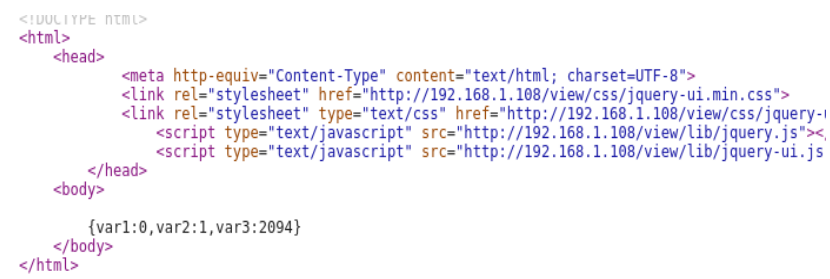

Figura 11: Resposta da requisição HTTP, escrita no node.

A variáveis que serão escritas nas saídas do node e que compõem a resposta da requisição HTTP devem estar entre as tags html <body> e $<$ /body $>$ respectivamente. Devem estar estruturados em formato json:

$\{\operatorname{var} 1: 0, \operatorname{var} 2: 1, \operatorname{var} 3: 2096\}$.

Onde var1,var2 e var3 serão respectivamente os valores das saídas digitais 1,2 
e da saída analógica. A figura 12 demonstra o fluxograma com todos os modos de operação.

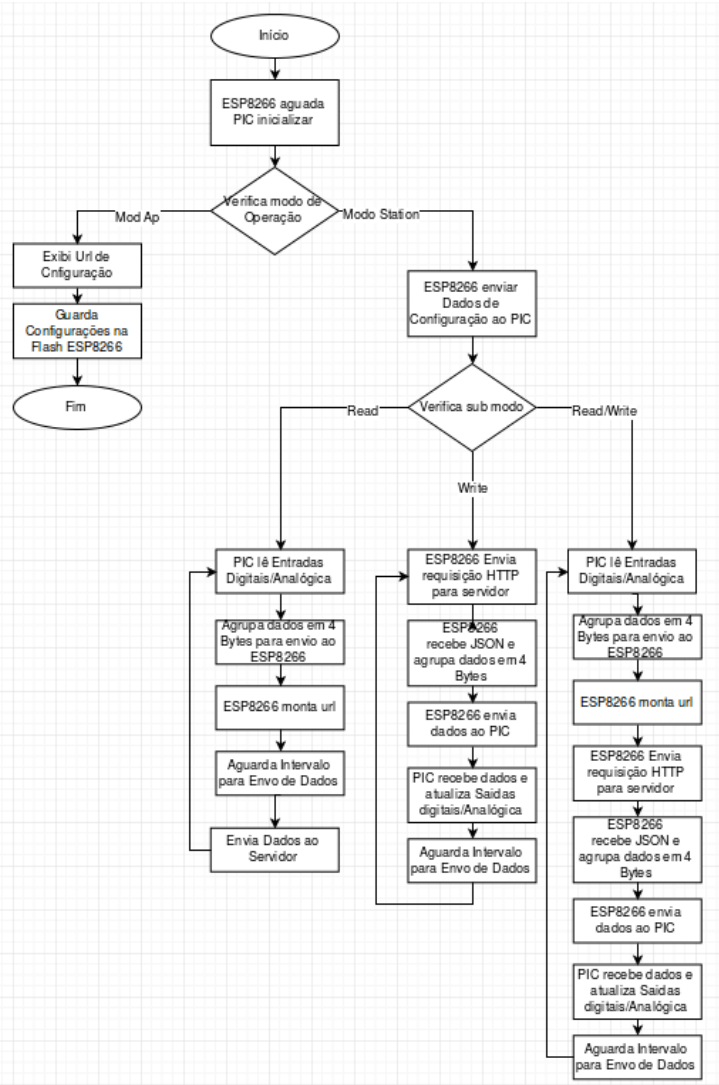

Figura 12: Fluxograma Modos de Operação

O que será feito com os dados lidos ou escritos depende do que será implementado no servidor, podendo ir desde uma simples lógica booleana como até um controlador PID, tudo integrado com aquisição e análise de dados de um CPS, proporcionando a um sistema legado a possibilidade de poder ter as características smart conection da arquitetura $5 \mathrm{C}$, podendo assim inserir nas indústrias antigas os benefícios da indústria 4.0.Na figura 13 temos a montagem final do protótipo.

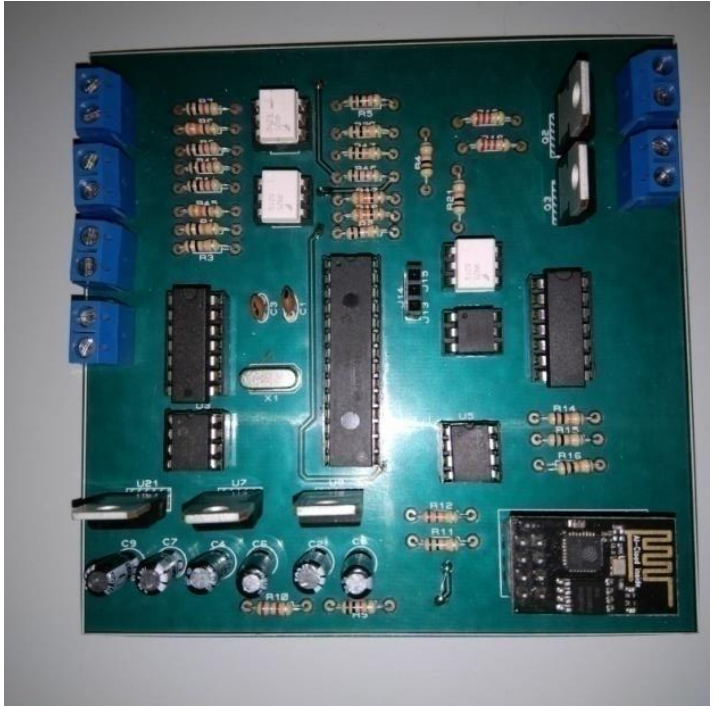

Figura 13: Protótipo NodeI4.0

\section{Conclusão e trabalhos futuros}

Este trabalho propôs a prototipação de um CPS que tem a capacidade de inserir qualquer sistema legado no nível smart conection da arquitetura $5 \mathrm{C}$, e com isso, poder conectar o mundo físico ao mudo cyber com a aquisição de dados confiáveis.

O protótipo foi testado em um sistema de controle de nível, medido por um transmissor de pressão e controlado por uma válvula proporcional. Nesse teste foi implementado um controlador proporcional no servidor.O NodeI4.0 se mostrou bastante eficiente com uma velocidade satisfatória para esse sistema, que não exige uma resposta abaixo de $50 \mathrm{~ms}$. Com o NodeI4.0, foi adicionado a esse simples sistema de controle as características de um CPS, descentralizado, permitindo a aquisição e manipulação dos dados e inserindo nas especificações da industria 4.0.

Para as próxima etapas do projeto será necessário a mudança do protocolo HTTP para protocolo customizado implementado em socket UDP, a fim de deixar o protótipo com uma baixa latência de comunicação e poder atender a sistemas que necessitam de maior velocidade.

Como implementações futuras integrar ao NodeI4.0 a possibilidade de subir mais um nível na arquitetura $5 \mathrm{C}$ passando assim a inferir informações a partir dos dados coletados. 


\section{Referências}

[1] Lasi, Heiner, et al. "Industry 4.0." Business \& Information Systems Engineering 6.4 (2014): 239.

[2] Festo. Industry 4.0 / IoT - Products and solutions.Disponível em:

https://www.festo.com/cms/nlbe_be/56644_56690.htm.Acesso em: 21 de Junho de 2017.

[3] Hermann, Mario, Tobias Pentek, and Boris Otto. Design Principles for Industrie 4.0 Scenarios. 2016 49th Hawaii International Conference on System Sciences (HICSS). IEEE, 2016.

[4] Jazdi, Nasser. "Cyber physical systems in the context of Industry 4.0." Automation, Quality and Testing, Robotics, 2014 IEEE International Conference on. IEEE, 2014.

[5] Lee, Jay, Behrad Bagheri, and Hung-An Kao. "A cyber-physical systems architecture for industry 4.0-based manufacturing systems." Manufacturing Letters 3 (2015): $18-23$

[6] Espressif, ESP8266. Disponível em :https://espressif.com/en/products/hardware /esp8266ex/overview.Acesso em: 21 de junho de 2017. 